Military Technical College Kobry El-Kobbah, Cairo, Egypt

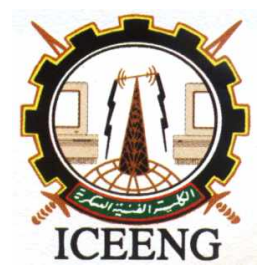

\title{
Channel estimation for OFDM systems using radial basis function networks
}

By

\author{
M.Nuri Seyman*
}

Necmi Taşpınar**

\section{$\underline{\text { Abstract: }}$}

In this paper, in order to estimate channel impulse responses in orthogonal frequency division multiplexing (OFDM), we use radial basis function network that is a kind of neural network, because of this structure is applicable of this kind of problem for its strong approximation and learning ability. We compare the performance of channel estimator based on radial basis function neural network with LS and MMSE algorithm with bit error rate (BER) and mean square error (MSE) criterias. Also Cramer Rao bound is given to evaluate the performances of estimators. Our proposal channel estimator has better performance than LS algorithm and closer performance to MMSE algorithm. However there is unnecessity of knowledge of channel statics and noise information of channel when neural structures are used as a channel estimator. Moreover after neural structures are trained, there is no need of sending pilot tones that are used to get channel impulse responses by LS and MMSE algorithm. As a result, system spendings are reduced.

\section{Keywords:}

Orthogonal frequency division multiplexing (OFDM), channel estimation, radial basis function network

* Department of Electronic Communication, Kırıkkale University, Kırıkale, Turkey

** Department of Electrical and Electronic Engineering, Erciyes University, Kayseri, Turkey 


\section{Introduction:}

Multicarrier modulation techniques such as orthogonal frequency division multiplexing (OFDM) are used in high data rate and quality communication systems. In OFDM, the wide-band spectrum is divided into multiple orthogonal narrow-band subcarriers. Each user's symbol is splitted into sub-symbols, each sub-symbol modulates a different subcarrrier and user sub-symbols are transmitted in parallel over these low-rate subcarriers. As a result of this transmission data can be transmitted in high rate and quality. In addition to these, multipath immunity and resistance to intersymbol interference (ISI) are other important advantages that OFDM has [1-2]. Digital audio broadcasting (DAB), digital video broadcastingterrestrial (DVB-T), digital subscriber line (DSL) and wireless local area network such as IEEE 802.11a and HIPERLAN/2 are the areas of communication systems that use OFDM as modulation scheme. However, to achieve coherent demodulation with high transmission rate, the receiver needs accurate channel impulse response (CIR) between receiver and transmitter. If channel estimation are not implemented, interchannel interference (ICI) occurs between adjacent sub-channels of OFDM. As a result of ICI, it is impossible to receive data by receiver correctly [3-7]. In order to estimate channel parameters at each subcarriers, least square (LS) and minimum mean square error (MMSE) algorithms can be used. In fast fading and time varying channels LS algorithm performs poorly in spite of implementation of this algorithms are quite easy. MMSE algorithm performs well in fast and dispersive fading channels but this algorithm requires noise characteristics of channel and channel statics to get CIRs [3]. In literature the channel estimators that use these algorithms are proposed to estimate channel informations. In [4], Edfords offer low rank channel estimation that is implemented to MMSE and compare this proposal with LS estimator using mean square error (MSE) and symbol error rate (SER) criterias. Also the performance of the LS and MMSE algorithm is compared in [5]. By considering these works it can be said that MMSE algorithm has better performance than LS algorithm. In [6-7], LS and MMSE are implemented for channel estimation in OFDM.

Besides classical techniques of channel estimation, heuristic approachs such as fuzzy logic and neural network are implemented to estimate and equalize channels for OFDM. In [8], fuzzy logic based channel estimator is applied to adjust the step size of LMS algorithm in OFDM and this algorithm can achieve robust tracking ability in various channel environments, faster convergence rate and low mean square error. In [9], a channel estimator including two parts of neural network is improved to estimate the amplitude and the angle of the frequency domain channel coefficients. Also neural networks are implemented to OFDM to solve channel equalization problem [10,11]. In [10] back propagation network that is a kind of learning algorithm of neural network is derived to equalize channels in OFDM. Furthermore RBFN equalizer which is trained to get channel responses by LMS algorithm 
structure is presented in [11].

In this paper, radial basis function network is used for channel estimation in OFDM. The performance of this estimator is compared with the classical LS and MMSE channel estimator with criterias of BER versus SNR and MSE versus SNR by computer simulations.

\section{OFDM System Model:}

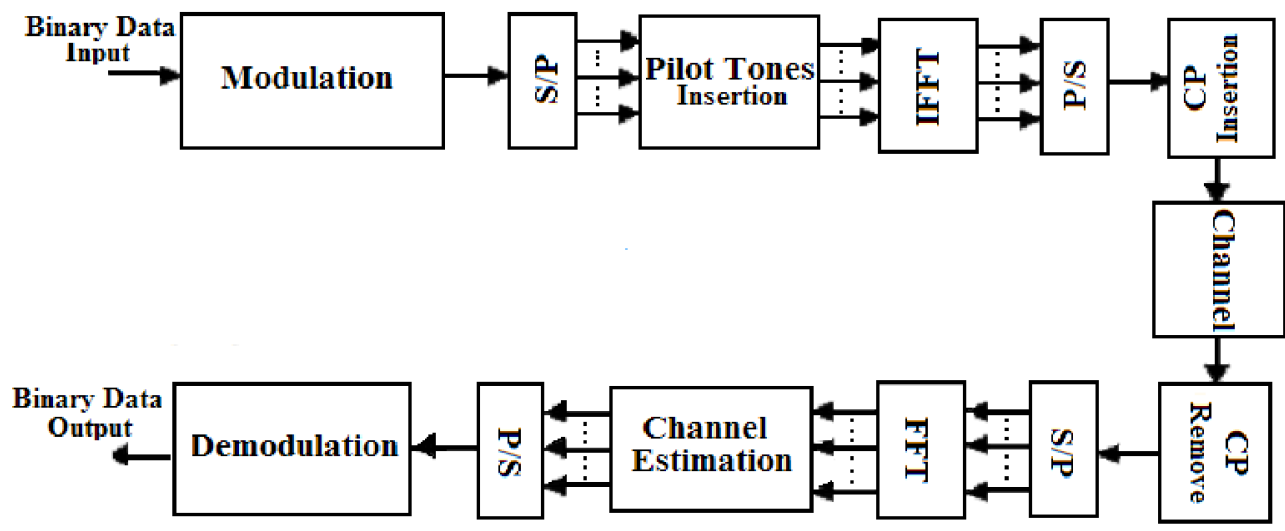

Figure (1):OFDM system model.

The block diagram of OFDM system is shown in Figure (1). To transmit data over wireless channels, serial binary data stream is mapped into phase signals. In serialparallel convertor, block data is converted to parallel. After insertion of pilot symbols that is used to get CIRs, modulated symbols $X_{m}(k)$ are transformed into time domain $\mathrm{x}_{\mathrm{m}}(\mathrm{n})$ signal:

$$
\begin{aligned}
\mathrm{x}_{\mathrm{m}}(\mathrm{n}) & =\operatorname{IFFT}\left\{\mathrm{X}_{\mathrm{m}}(\mathrm{k})\right\} \\
& =\sum_{\mathrm{k}=0}^{\mathrm{N}-1} \mathrm{X}_{\mathrm{m}}(\mathrm{k}) \mathrm{e}^{\mathrm{j}(2 \pi \mathrm{n} / \mathrm{N})} \quad \mathrm{n}=0, \ldots, \mathrm{N}-1
\end{aligned}
$$

Cyclic prefix (CP) is inserted to prevent intersymbol interference (ISI) and resultant samples are:

$$
x(f)=\left\{\begin{array}{cc}
x(N+n) & n=-N_{G}, \ldots ., 1 \\
x(n) & n=0,1, \ldots, N-1
\end{array}\right.
$$

Then signal is transmitted over wireless channel. The received signal is given by 


$$
\mathrm{y}_{\mathrm{f}}(\mathrm{n})=\mathrm{x}_{\mathrm{f}}(\mathrm{n}) \otimes \mathrm{h}(\mathrm{n})+\mathrm{w}(\mathrm{n})
$$

When signal is transmitted over frequency selective multipath fading channels, the channel impulse responses $h(n)$ can be expressed as

$$
\mathrm{h}(\mathrm{n})=\sum_{1=0}^{\mathrm{L}-1} \alpha_{1} \delta\left(\mathrm{n}-\tau_{1}\right)
$$

After removing CP from $\mathrm{y}_{\mathrm{f}}(\mathrm{n}), \mathrm{y}(\mathrm{n})$ is received. Then FFT is taken as

$$
\mathrm{Y}(\mathrm{k})=\operatorname{DFT}\{\mathrm{y}(\mathrm{n})\}=\frac{1}{\mathrm{~N}} \sum_{\mathrm{n}=0}^{\mathrm{N}-1} \mathrm{y}(\mathrm{n}) \mathrm{e}^{-\mathrm{j} 2 \pi \mathrm{kn} / \mathrm{N}} \quad \mathrm{k}=0,1, \ldots, \mathrm{N}-1
$$

Then the signal is represented by

$$
\mathrm{Y}(\mathrm{k})=\mathrm{X}(\mathrm{k}) \mathrm{H}(\mathrm{k})+\mathrm{W}(\mathrm{k})
$$

\section{Radial Basis Function Networks for Channel Estimation:}

Artificial neural networks are nonlinear information processing devices that are built from interconnected elementary processing devices called neurons. Self organization, adaptation, universal approximation and learning capabilities are the most important properties of the neural networks. Radial basis function (RBF) network is a kind of neural networks. Radial basis functions are embedded into a two layer feed forward neural network. Such a network is characterized by a set of inputs and set of outputs. Between inputs and outputs there is a layer proccesing units called hidden units. Each of them implements a radial basis function. The output layer implements a weighted sum of hidden unit outputs. The function of these nets is given by:

$$
\mathrm{y}_{\mathrm{i}}(\mathrm{x})=\sum_{\mathrm{j}=1}^{\mathrm{n}} \mu_{\mathrm{i}, \mathrm{j}} \phi\left(\mathrm{x}-\mathrm{x}_{\mathrm{j}}\right)
$$

where $\phi\left(x-x_{j}\right)$ is the activity of the hidden node $\mathrm{j}$, with a RBF function centered on the vector $x_{j}$. In hidden layer, gaussian function which is selected as activation function generally is follows;

$$
\phi_{j}\left(x-x_{j}\right)=\exp \left(-\frac{\sum_{k=1}^{K}\left(x_{k}-\mu_{j, k}\right)^{2}}{2 \sigma^{2}}\right)
$$


The weights $\mu_{j, k}$ between node $\mathrm{k}$ in the input layer and node $\mathrm{j}$ in the hidden layer do not act multiplicatively as in other neuron models, but define the input vector $\mathrm{x}_{\mathrm{j}}=\left(\mu_{\mathrm{j} 1}, \ldots, \mu_{\mathrm{jK}}\right)$ eliciting the maximum response of node $\mathrm{j}$ [12]. In the learning procedure, the weights in hidden layer are updated.

\subsection{Channel Estimation Using Radial Basis Function Network}

To train network we used correct channel impulse responses that got using pilot tones. Since the OFDM symbols consist of complex signals whether neural network uses real signals, in order to adopt the neural network to OFDM, each complex signals are seperated into real and imaginary parts. Then the seperated signals which is carried by each subcarriers are inputted to network as seen in Figure (2).

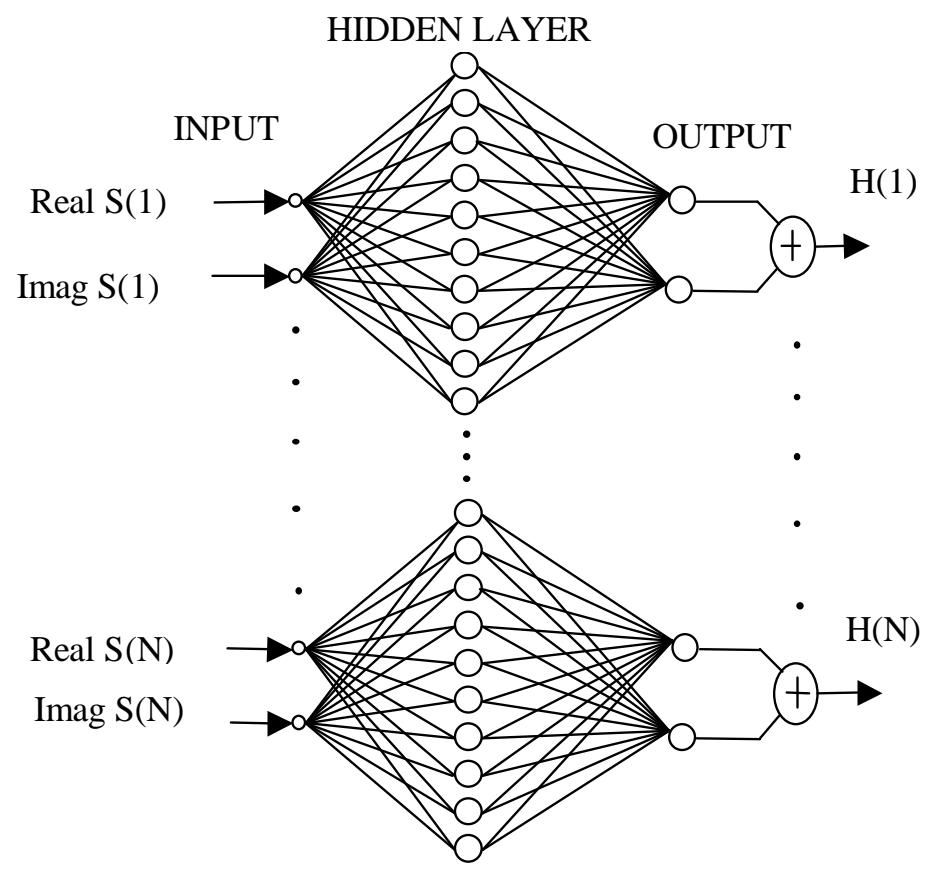

Figure (2): Radial basis function structure for channel estimation.

Gauss function was used for activition in our simulations. The $i_{\text {th }}$ output layers of our networks are:

$$
\begin{aligned}
& \mathrm{H}_{\text {ireal }}(\mathrm{k})=\sum_{\mathrm{j}=1}^{\mathrm{n}} \mu_{\mathrm{i}, \mathrm{j}} \phi\left(\mathrm{S}_{\text {real }}(\mathrm{k})-\mathrm{S}_{\text {jreal }}(\mathrm{k})\right) \\
& \mathrm{H}_{\text {iimag }}(\mathrm{k})=\sum_{\mathrm{j}=1}^{\mathrm{n}} \mu_{\mathrm{i}, \mathrm{j}} \phi\left(\mathrm{S}_{\text {imag }}(\mathrm{k})-\mathrm{S}_{\text {jimag }}(\mathrm{k})\right)
\end{aligned}
$$




\section{Simulation Results:}

The performance of channel estimator that uses radial basis function network is compared with LS and MMSE channel estimators with bit error rate (BER) and mean square error (MSE) versus signal to noise ratios (SNR) criterias in our simulations. In Table (1), the simulation parameters that we used in our simulations are shown.

Table(1): OFDM system parameters

\begin{tabular}{|l|c|}
\hline \multicolumn{1}{|c|}{ Parameters } & Values \\
\hline Carrier frequency $\left(f_{c}\right)$ & $5 \mathrm{GHz}$ \\
\hline Bandwidht & $20 \mathrm{MHz}$ \\
\hline FFT size & 64 \\
\hline Number of subcarriers & 54 \\
\hline Cyclic prefix size & 16 \\
\hline Modulation type & QPSK \\
\hline
\end{tabular}

QPSK modulation type is used over 6 ray multipath fading with $[0,200,300,500$, $1000,1500]$ ns relative delays and $[-3,0,-2,-7,-10,-12] \mathrm{dB}$ power paths channel to transmit data.

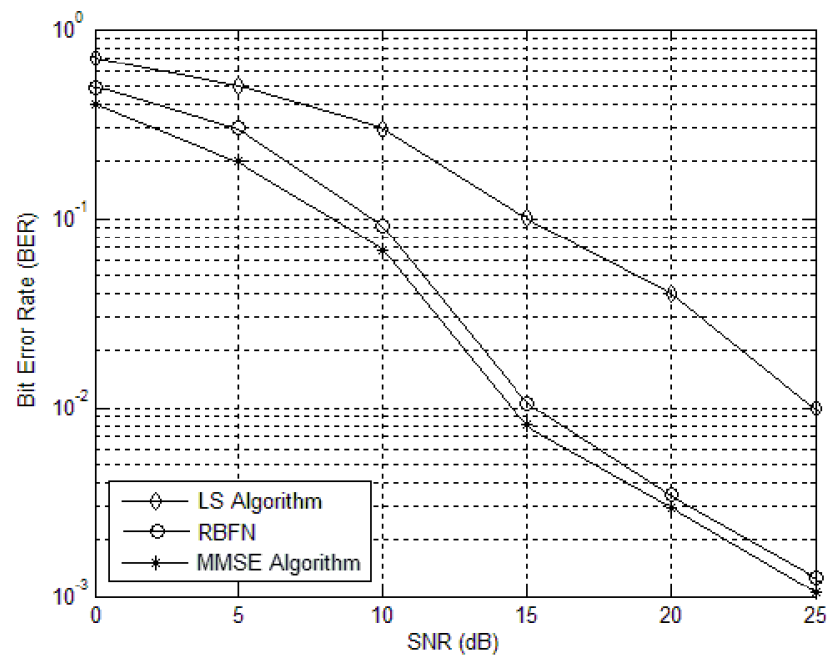

Figure(3):BER values of the channel estimators versus SNR.

Signal to noise ratio at $0-25 \mathrm{~dB}$ interval versus bit error rate for channel estimator are shown in Figure (3). According to this figure, not only at the low SNR but also at the high SNR values, peformance of RBF neural estimator is better than LS algorithm. Especially at $20 \mathrm{~dB}$ SNR value the differences of bit error rate between LS and RBF neural is more than $10^{-1}$. And bit error rates of RBF neural is very close to MMSE's. 
At increased SNR values RBF neural and MMSE have almost the same bit error rate.

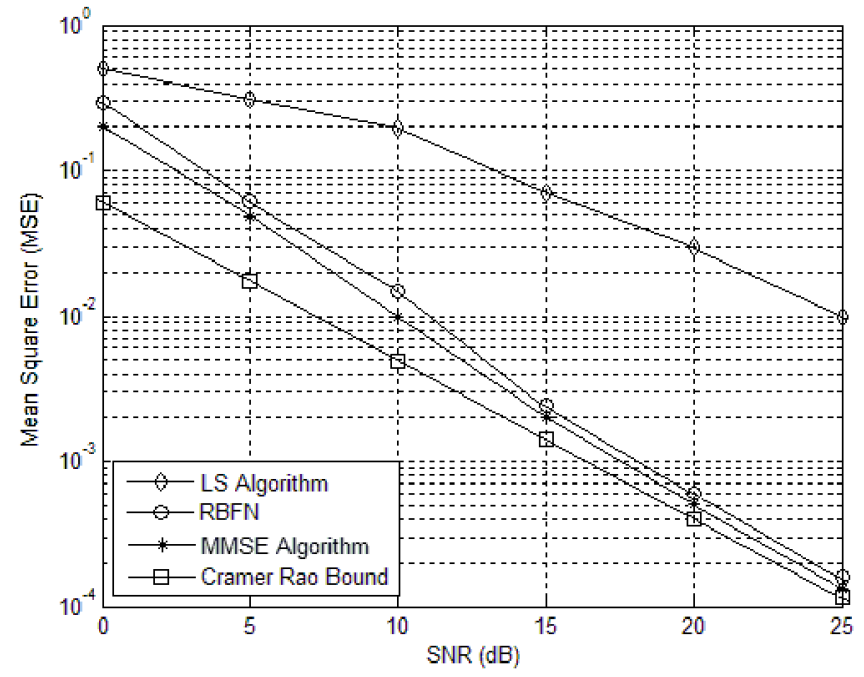

Figure(4):MSE values of the channel estimator versus SNR.

In Figure (4), mean square errors of channel estimators are shown for another performance evaluation. Also Cramer Rao bound is given in order to evaluate the performance of estimators. The formulation in [13] is used to compute the Cramer Rao bound versus SNR. As it is shown in Figure (4), at each SNR values, estimation errors of LS algorithm is more than other estimators. MSE of MMSE algorithm is close to Cramer Rao bound at $15 \mathrm{~dB}$ SNR value considerably. And MSE of neural network is very close to Cramer Rao bound as well as MMSE algorithm.

\section{Conclusion:}

In this paper, radial basis function network was used to solve the channel estimation problem for OFDM systems. To demonstrate the performance of RBF network channel estimator, it was compared with classical estimation algorithms such as LS and MMSE. According to the simulation results, the performance of trained radial basis function network is better than LS's and quite close to MMSE's. Although MMSE is the algorithm that has the best performance and very close to Cramer Rao bound among the channel estimation algorithms that we simulated, it is more complex than others, because it estimates channel impulse responses based on channel statics and noise information. Furthermore MMSE algorithm needs the matrix inverse and correlation computation. But RBF neural estimator does not require channel statics and noise information to get channel impulse responses and it has low complexity than MMSE. Unnecessity of pilot tone transmission is another advantage of the RBF neural network channel estimator for OFDM. 


\section{References:}

[1] L. J., Cimini, Analysis and Simulation of Digital Mobile Channel Using Orthogonal Frequency Division Multiplexing, IEEE Transactions on Commununication, Vol. 33, No.7, P. 665-675, 1985.

[2] R. Van Nee, R. Prasad, OFDM for Wireless Multimedia Communications, Artech House, London, Publishers, 2000.

[3] S. Coleri, M. Ergen, A. Puri, A. Bahai, Channel Estimation Techniques Based on Pilot Arragement in OFDM Systems, IEEE Transaction on Broadcasting, Vol. 48, No. 3, P. 223-229, 2002.

[4] J.-J. Van de Beek, O. Edfords, M. Sandell, S.K. Wilson, and. P.O. Borjesson, On Channel Estimation in OFDM Systems, Proceeding of the IEEE 45th Vehicular Technology Conference, P. 815-819, Chicago, July, 1995.

[5] O. Edford, M. Sandell, J.J. Beck, S.K.Wilson, P.O. Börjesson, OFDM Channel Estimation by Using Singular Value Decomposition, IEEE Transaction on Communication, Vol 46, No.7, P. 931-939, 1998

[6] M. X. Chang, A New Derivation of Least Squares Fitting Principle for OFDM Channel Estimation, IEEE Transaction on Commununication, Vol. 5, No. 4, P. 726-731, 2006.

[7] V. Srivastava, C. K. Ho, P. H. Fung, S. Sun, Robust MMSE Channel Estimation in OFDM Systems with Practical Timing Synchronization, Proceedings of the IEEE Wireless Communications and Networking, P. 711-716, Atlanta, 2004.

[8] J.H. Wen, C. Chang, Y. Lee, C. Y. Huang, OFDM Channel Prediction Using Fuzzy Update LMS Algorithm in Time-Variant Mobile Channel, Proceedings of the IEEE 64th Vehicular Technology Conference, P. 1-5, Montreal, Canada, September 2006.

[9] J. Sun, D.F. Yuan, Neural Network Channel Estimation Based on Least Error Algorithm in the OFDM Systems, Lecture Notes in Computer Science, P. 706711, 2006.

[10] E. Chen, R. Tao, X. Zhao, Channel Equalization for OFDM System Based on the BP Neural Network, Proceeding of the 8th IEEE International Conference on Signal Processing, Vol.3, Beijing, 2006. 
[11] G. Charalabopoulupos, P. Stavroulakis, A.H. Aghvami, A Frequency-Domain Neural Network Equalizer for OFDM, Proceeding of the IEEE Global Communication Conference, P. 571-575, San Francisco, 2003.

[12] M. J. D. Powell, Radial Basis Functions for Multivariate Interpolation:A review, Algorithms for Approximation, Clarendon Press, Oxford, 1987.

[13] O. Simione, Y. Bar-Ness, U. Spagnolini, Pilot Based Channel Estimation for OFDM Systems by Tracking the Delay Subspace, IEEE Transaction on Wireless Commununication, Vol. 3, No. 1, P. 315-325, 2004.

\section{Nomenclatures:}

N... Number of subcarriers

$\mathrm{N}_{\mathrm{G}} \ldots$ Number of the samples in cyclic prefix interval

$\mathrm{h}(\mathrm{n})$... Channel impulse response in time domain

$\mathrm{w}(\mathrm{n}) \ldots$ White gaussian noise

$\alpha_{1} \ldots$ Time variant complex path gain of $1_{\text {th }}$ path

$\tau_{1} \ldots$ Delay of the $1_{\text {th }}$ path

$y_{i} \ldots$ Activity of the output node $i$ of RBFN,

$\sigma \ldots$ Width of gaussian function

K... Dimension of the neural input space

X... Actual input vector to the neural network

$\mu_{\mathrm{ij}} \ldots$ Weights from the RBF nodes in the hidden layer to linear output node 